\title{
Aplicación de un modelo Lean-BIM para la mejora de la productividad en redacción de proyectos de edificación
}

\section{Application of Lean-BIM model to improve design building phase's productivity}

\author{
$\underline{\text { A. Latorre Uriz }}^{(*)}$, C. Sanz ${ }^{(* *)}$, B. Sánchez ${ }^{(* * *)}$
}

\section{RESUMEN}

El presente trabajo propone un modelo para la mejora del rendimiento de la redacción de proyectos de edificación en BIM. La productividad del sector de la construcción es una materia que preocupa y genera debates acerca del modelo de producción adecuado para mejorar su rendimiento. Si se compara el nivel de productividad de la construcción con otros sectores, tales como la industria o el automóvil, resulta bajo, y la evolución de los últimos años evidencia un incremento de esta diferencia.

Se presenta un modelo que combina el uso de la filosofía Lean mediante la aplicación de Lean Construction, y la metodología BIM, fruto de una revisión bibliográfica y la investigación realizada por los autores. El modelo se testa en 6 casos de estudios, para posteriormente ser validado mediante técnicas cualitativas y cuantitativas.

Palabras clave: BIM, gestión, Lean Construction, productividad.

\section{ABSTRACT}

This paper proposes a model to improve the design phase's performance of construction projects developed with BIM. Construction industry's productivity is an issue that concerned and provoke debates about the production model suitable for improve their performance. If construction productivity level is compared with others industries, such as industry or automobile, is low, and the evolution of recent years shows an increase in this difference.

A model is presented, that combines the use of Lean Construction and BIM methodology. This model is result of a literature review and the research carried out by the authors. The model is tested in 6 cases studies, and it's validated by qualitative and quantitative techniques.

Keywords: BIM, Lean Construction, management, productivity.

(*) Estudiante de doctorado, Departamento de Construcción, Instalaciones y Estructuras de la Escuela Técnica Superior de la Universidad de Navarra (España).

(**) Profesora Titular, Departamento de Construcción, Instalaciones y Estructuras de la Escuela Técnica Superior de la Universidad de Navarra (España).

$\left(^{* * *}\right)$ Profesor Asistente, Departamento de Construcción, Instalaciones y Estructuras de la Escuela Técnica Superior de la Universidad de Navarra (España).

Persona de contacto/Corresponding author: alatorre@alumni.unav.es (A. Latorre Uriz).

ORCID: http://orcid.org/oooo-0oo2-3679-7029 (A. Latorre Uriz); https://orcid.org/oooo-0oo1-6788-5315 (C. Sanz); https://orcid.org/oooo-0oo1-6808-3314 (B. Sánchez)

Cómo citar este artículo/Citation: Latorre Uriz, A.; Sanz, C.; Sánchez, B. (2019). Aplicación de un modelo Lean-BIM para la mejora de la productividad en redacción de proyectos de edificación. Informes de la Construcción, 71(556): e313. https://doi.org/10.3989/ic.67222.

Copyright: (c) 2019 CSIC. Este es un artículo de acceso abierto distribuido bajo los términos de la licencia de uso y distribución Creative Commons Reconocimiento 4.0 Internacional (CC BY 4.0). 


\section{INTRODUCCIÓN}

La productividad es un tema que preocupa al sector de la construcción y que ha generado múltiples debates acerca de cuál debería ser el modelo de producción adecuado. En una encuesta realizada a más de 270 profesionales del sector en España, un 70\% considera necesaria una mejora de la productividad, calificándola como "negativa" o "muy negativa". Un $91 \%$ considera necesario para lograr la mejora un cambio en la gestión de los proyectos, valorando como positiva la adopción de técnicas del sector industrial un 80\% de ellos. Dentro de este contexto, el uso de Building Information Modelling (BIM) como principal herramienta de digitalización, bajo el enfoque de la filosofía Lean Construction (LC), se plantea como una solución a los problemas de productividad del sector.

La implantación de BIM en Europa ha experimentado un crecimiento exponencial en los últimos años, a partir de la entrada en vigor de la Directiva Europea sobre Contratación Pública de 2014. Los países nórdicos y otros, como EEUU, Nueva Zelanda o Singapur, llevan utilizando BIM de manera generalizada desde la década del 2000. El uso de BIM conlleva una mejora del flujo de comunicación, coordinando mejor la secuencia de trabajo y aportando mayor visualización para mejorar la comprensión del proyecto, mientras que la aplicación de LC, menos extendida a día de hoy que BIM, aporta mejoras como una mayor entrega de valor y calidad al cliente y la mejora de la productividad y la gestión de todo el proceso.

Ambas metodologías dan una gran importancia a la colaboración entre los agentes participantes en el proyecto, tal y como reflejan diferentes autores (1-5). LC es una filosofía orientada a la creación de valor para el cliente, optimizando al máximo los recursos; BIM se centra en la aplicación de tecnologías que posibilitan y facilitan el incremento del grado de colaboración entre todos los agentes participantes (2).

LC permite involucrar y hacer partícipes a todos los agentes del proceso en un estadio más temprano y los modelos BIM aportan una gran capacidad de documentar y registrar la información que contienen. El incremento de la colaboración entre los agentes implicados que de ello se deriva es muy significativo, ya que en los proyectos desarrollados mediante el método tradicional Proyecto-Adjudicación-Ejecución alrededor de un $80 \%$ de las reclamaciones son formuladas por los agentes intervinientes, siendo el $87 \%$ de ellas solicitudes de más información, de cambios por falta de información o de cambios o modificaciones (6).

Los proyectos involucran cada vez a un número mayor de agentes pertenecientes a diferentes empresas. El reto del sector es que todos persigan un mismo objetivo, lo que en la actualidad no sucede ya que los contratos tradicionales favorecen que cada empresa mire sólo por su propio interés (7). De ello se derivan numerosas modificaciones de proyecto, uno de los mayores desperdicios que se genera en la fase de redacción del mismo (8). La necesidad de disminuir los desperdicios de la construcción es imperiosa (9), ya que se estima que el 49,7\% del tiempo empleado en los proyectos no genera valor (8). Cuanto más compleja es la redacción de un proyecto y su posterior ejecución, menor es la productividad (10). Para solventarlo deben utilizarse nuevas tecnologías que posibiliten disminuir tanto el coste de la redacción del proyecto como su posterior ejecución, a la vez que la mejora de los procesos (11), pero su implantación implica vencer la resistencia al cambio que caracteriza al sector de la construcción, uno de los más reacios a ello (12).

Mejorar la productividad implica la mejora de los procesos mediante el empleo de nuevas tecnologías y procedimientos constructivos (13), ya que una correcta gestión de la construcción requiere de la integración de procesos, tecnología y personas para lograr alcanzar sus objetivos (9). Se necesitan nuevas teorías de producción para mejorar esta situación, y la aplicación conjunta de LC y BIM que se propone puede ser una posible solución (10), por cuanto constituyen dos enfoques prometedores para la mejora de la productividad del sector $(13,14)$. Conceptos nuevos, que se han ido desarrollando por separado, sin llegar a ser comprendidos del todo, por lo que no se ha logrado mejorar todo lo posible los procesos (15). Se han hecho implantaciones independientes, pero no hay muchos ejemplos de su aplicación conjunta, no existiendo una visión única que permita potenciar al máximo sus cualidades individuales (15). Para la implantación conjunta, lógicamente es esencial tener un amplio conocimiento de cada uno por separado (13).

En el momento actual, la mejora real y efectiva de la productividad en construcción exige afrontar cambios tecnológicos y transformar los procesos. La aplicación conjunta de LC y BIM constituye una solución viable a los problemas que, por falta de colaboración y escaso rendimiento, sufre el sector.

\subsection{Vinculación entre Lean Construction y BIM}

La investigación sobre la vinculación entre LC y BIM se ha desarrollado principalmente desde 2006, año en el que se identifican referencias conjuntas de ambas metodologías (2): Rischmoller et al (16) evaluaron bajo un enfoque Lean el impacto de lo que denominaron Herramientas Informáticas Avanzadas para la Visualización (Computer Aided Visualization Tools, CAVT), destacando las sinergias entre ambas, y Khanzode et al (17) relacionaron el uso del Lean Project Delivery System (LPDS) con el Virtual Design and Construction (VDC), concluyendo que el uso conjunto de LC y BIM resulta beneficioso, obteniéndose mejores resultados.

Entre 2006 y 2012, se desarrollan trabajos en los que se establece un marco teórico de la vinculación entre ambos conceptos $(2,18,19,20,21,22,23)$. Uno de los más importantes es el de Sacks et al (2), que aporta una matriz que contiene interacciones entre LC y BIM. En 2012, en el $20^{\circ}$ Congreso Internacional de Lean Construction, se analizaron las interacciones entre LC y BIM halladas en un caso de estudio (3), y se integró el uso de BIM junto a la técnica del LPS (1).

En 2013, Dave (24) desarrolló el vínculo entre LC y BIM, incluyendo el software Visilean que facilita el empleo conjunto de LC y BIM.

En los siguientes años, destaca el desarrollo de la vinculación LC-BIM mediante el estudio de casos reales de implantación o el desarrollo de marcos teóricos enfocados exclusivamente a la fase de ejecución del proyecto $(9,10,11,13,14,25,26)$, en los que principalmente se analizan los beneficios de la visual- 
ización que aporta el modelo BIM y se vincula la planificación mediante el uso del Last Planner System (LPS) a las simulaciones $4 \mathrm{D}$ del mismo.

El interés suscitado por la relación entre LC y BIM se ha ido incrementando durante la última década, adquiriendo cada vez mayor relevancia. Sin embargo, hasta el momento, la investigación se ha centrado en destacar más las conexiones existentes entre LC y BIM que en desarrollar una metodología de trabajo conjunta (27). Se han realizado diversos estudios sobre la implantación conjunta centrada en la fase de ejecución, excluyendo la fase de redacción del proyecto, que no se ha desarrollado de la misma manera. En los últimos años, se han realizado también análisis teóricos sobre las ventajas que puede aportar el uso conjunto de LC y BIM en fases tempranas $(7,8,28,29)$, pero no hay estudios sobre casos reales en los que se comprueben las mejoras conseguidas. Hay un interés creciente en el uso combinado de Lean y BIM pero la mayoría de las empresas del sector se encuentran todavía en un estadio muy inicial con respecto a la implementación conjunta (30).

\subsection{Interacción Lean Construction y BIM}

En los estudios realizados por diferentes autores en los últimos años, se han identificado diversos puntos de interacción entre LC y BIM. La productividad del sector puede mejorar con el uso de ambas, pero ello exige también un cambio de mentalidad y de la manera de trabajar; cambio de mentalidad como el que posibilita la filosofía Lean (7), siendo BIM una de las herramientas más importantes para la consecución de los objetivos perseguidos (11). Con el uso de modelos BIM y la metodología que ello conlleva, se favorecen los procesos de la filosofía Lean (25).

Las principales interacciones entre LC y BIM se han analizado considerando la vinculación existente entre los principios Lean y las aplicaciones BIM $(7,9,25)$. (Tabla 1$)$.

Tabla 1. Interacción entre los Principios Lean y las Aplicaciones BIM con las que se relacionan.

\begin{tabular}{|l|l|}
\hline \multicolumn{1}{|c|}{ Principios Lean } & \multicolumn{1}{c|}{ Aplicaciones BIM } \\
\hline Mejora del flujo de trabajo & Visualización del modelo \\
\hline Mejora de la calidad & Simulación constructiva/4D \\
\hline Reducción carga de trabajo & Detección interferencias en el modelo \\
\hline Generación de Valor & Generación de alternativas \\
\hline Ahorro tiempos & Colaboración agentes implicados \\
\hline Reducción de pérdidas & Transparencia \\
\hline
\end{tabular}

De entre los trabajos realizados, destaca el ya citado de Sacks et al (2), del que resulta una matriz de 18x24 con 56 puntos de interacción entre los principios de LC y las principales aplicaciones de BIM. Los principios Lean con más interacciones coinciden con los identificados por otros autores: Reducir la variabilidad (que repercute en una mejora del flujo de trabajo y la calidad), reducir tiempos y generar valor. En cuanto a las aplicaciones BIM que destacan también coinciden con las analizadas: Visualización, uso del modelo para el análisis, búsqueda de una mejor colaboración y generación de alternativas.

Por lo tanto, el trabajo teórico previo de otros autores, permite identificar suficientes puntos de interacción entre LC y BIM como para avanzar que su aplicación conjunta supone una mejora del rendimiento de ambas, haciendo hincapié en la idea de que el marco contextual del cambio de paradigma del sector debe basarse en la filosofía Lean mediante la aplicación de LC, y apoyarse en BIM como principal herramienta para el cambio.

\subsection{Mejoras derivadas del uso de lean construction y BIM}

LC y BIM no son dependientes uno del otro, pudiendo implantarse por separado (2). No obstante, los beneficios de aplicar BIM con un enfoque Lean aumentan al potenciarse los aspectos positivos. Para alcanzar su potencial completo, se necesitan mutuamente (20). Además, si ambos se combinan con el método de desarrollo de proyecto Integrated Project Delivery (IPD), se potencian más aún las mejoras, al fomentarse todavía más la colaboración entre agentes, al hacer partícipes a todos los involucrados en las fases iniciales del desarrollo proyecto $(10,21)$.

En la tabla 2 figuran las principales mejoras derivadas el uso combinado de LC y BIM.

Tabla 2 Mejoras derivadas del uso de LC - BIM.

\begin{tabular}{|l|c|}
\hline \multicolumn{1}{|c|}{ Mejora } & Autor \\
\hline Disminución de re-trabajos & $(28,25,22)$ \\
\hline Una única fuente de información de proyecto & $(28)$ \\
\hline Mayor integración del proyecto & $(14,10,23)$ \\
\hline Mejora de la productividad & $(22,8,9,11)$ \\
\hline Aumento de la colaboración & $(25,7,11,21)$ \\
\hline $\begin{array}{l}\text { Disminución del tiempo de ciclo de vida del } \\
\text { proyecto }\end{array}$ & $(25,10,9,21)$ \\
\hline Aumento del valor generado/entregado & $(25,22,23,11,10)$ \\
\hline Aumento de la visualización & $(9,21,7)$ \\
\hline Disminución de conflictos & $(11,21,7)$ \\
\hline Reducción de tiempos & $(11)$ \\
\hline Eliminación de pérdidas & $(22,11)$ \\
\hline
\end{tabular}

Como cabía esperar, las principales mejoras derivadas del uso combinado de LC y BIM se relacionan claramente con las interacciones identificadas en la matriz de Sacks (2). Principios Lean tales como la entrega de valor al cliente, la mejora del flujo o la mejora continua, junto a aplicaciones BIM como la visualización, el aumento de la colaboración o el análisis de los proyectos, son las principales causas de estas mejoras.

\section{METODOLOGía}

El objetivo principal del presente artículo es describir la investigación realizada para elaborar el modelo propuesto de aplicación conjunta de LC y BIM para la fase de redacción del proyecto. En la investigación se establecieron los siguientes objetivos parciales: (1) Identificar los principales nexos de unión entre LC y BIM, (2) Realizar un primer modelo que combinara su uso, (3) Testar el modelo en casos reales, y (4) Realizar mejoras al modelo inicialmente propuesto.

La metodología investigadora (Figura 1) se dividió en tres fases: Una primera etapa, basada en una revisión bibliográfica para identificar los principales vínculos entre LC y BIM; una segunda fase donde se realizó una propuesta de modelo y se testó mediante 6 casos de estudio; y una tercera fase en la que se realizaron una validación del modelo a través de la realización de entrevistas y cuestionarios. 
Fase de Exploración

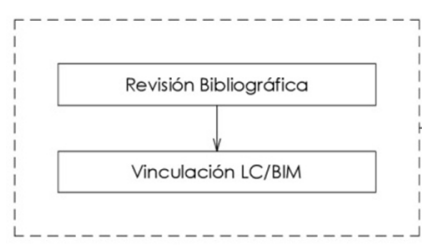

Fase de modelado

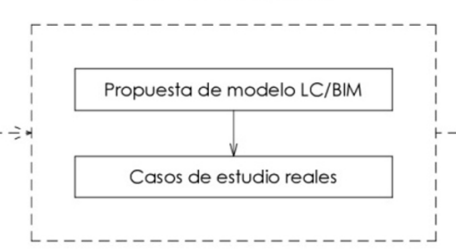

Fase de validación

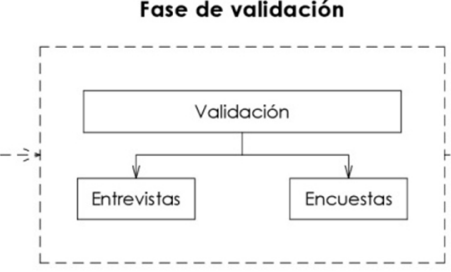

Resultado

Modelo Lean Construction-BIM

Figura 1. Metodología Investigadora.

\section{CASO DE ESTUDIO}

En este apartado se expone como se ha comprobado que todos los beneficios identificados de manera lógica y teórica por diversos autores como conseguidos tras la aplicación real de BIM bajo un enfoque Lean durante la ejecución de proyectos de edificación, se consiguen también durante la fase de proyecto. El estudio se ha centrado en proyectos de edificación, seleccionando una empresa tipo del sector en cuanto a tamaño y facturación, y analizando comparativamente 3 proyectos desarrollados con el flujo de trabajo original de la empresa, con otros 3 proyectos realizados con el derivado de la nueva propuesta de modelo. Para ello fue necesario analizar previamente el flujo de trabajo original de la empresa. Señalar que los autores participaron en los 6 proyectos estudiados.

Para comprobar si de dicha modificación se derivaba una mejora de la productividad, se compararon los 6 proyectos realizados por la empresa, tres con el flujo original y tres con el nuevo flujo modificado propuesto, para conseguir datos objetivos que permitieran evaluar si el cambio del procedimiento de trabajo suponía o no una mejora real para la empresa.

Se establecieron hasta 13 indicadores diferentes, divididos en 3 grupos (Tabla 3 ).

\subsection{Análisis del flujo original}

La empresa es una oficina técnica de arquitectura con sede en Pamplona, especializada en la realización de proyectos de edificación, con un nivel inicial de implementación de BIM. La empresa abarca todo el proceso edificatorio, desde la comercialización y redacción del proyecto hasta el seguimiento del proceso de ejecución, tanto en proyectos de obras de nueva planta como de rehabilitación de edificios existentes.

En el flujo original de trabajo de la empresa se identificaron una serie de problemas y aspectos de mejora potenciales, que se recogen en las tablas 4 y 5 respectivamente.

Tabla 4. Problemas identificados en el flujo de trabajo.

\begin{tabular}{|l|}
\hline \multicolumn{1}{|c|}{ Problemas identificados } \\
\hline - La no presencia de los responsables del proyecto en materia \\
de modelado en la reunión previa provoca dudas en fases \\
posteriores que generan retrasos.
\end{tabular}

Tabla 3. Indicadores del rendimiento del proyecto.

\begin{tabular}{|c|c|}
\hline \multicolumn{2}{|r|}{ Indicadores de proyecto } \\
\hline H/Modelado & Cantidad de tiempo destinado a modelar elementos nuevos. \\
\hline H/Cambios modelo & Cantidad de tiempo destinado a modifica elementos ya modelados \\
\hline $\begin{array}{l}\text { H/CTA (Comportamiento Térmico } \\
\text { Acústico) }\end{array}$ & $\begin{array}{l}\text { Cantidad de tiempo destinado al estudio del comportamiento térmico } \\
\text { y acústico del proyecto. }\end{array}$ \\
\hline $\mathrm{H} / \mathrm{Planos}$ & $\begin{array}{l}\text { Cantidad de tiempo destinado a la maquetación y montaje de planos } \\
\text { del proyecto. }\end{array}$ \\
\hline $\begin{array}{l}\text { H/Memoria (Documentación escrita, } \\
\text { memoria, anejos calculo, pliego, } \\
\text { menos Mediciones y Presupuestos) }\end{array}$ & $\begin{array}{l}\text { Cantidad de tiempo destinado a la redacción de la documentación } \\
\text { del proyecto. }\end{array}$ \\
\hline $\mathrm{H} /$ Cambios & $\begin{array}{l}\text { Cantidad de tiempo destinado a la modificación de planos o de la } \\
\text { memoria, a la parte de documentación del proyecto. }\end{array}$ \\
\hline \multicolumn{2}{|r|}{ Indicadores de modelado } \\
\hline \%new & Porcentaje de creación de nuevos elementos del modelo. \\
\hline \%mod & Porcentaje de modificación de elementos del modelo. \\
\hline$\%$ doc & $\begin{array}{l}\text { Porcentaje de actividades destinadas a montaje de planos, tablas y } \\
\text { completar información del proyecto. }\end{array}$ \\
\hline$\%$ rev & Porcentaje de actividades destinadas a la revisión del modelo. \\
\hline$\%$ snc & Porcentaje de sincronizaciones debido a conflictos durante el modelado. \\
\hline \multicolumn{2}{|r|}{ Indicadores generales de proyecto } \\
\hline h/totales & Cantidad de tiempo invertido en el proyecto \\
\hline $\mathrm{h} / €$ & $\begin{array}{l}\text { Ratio que relaciona las horas invertidas en el proyecto con su } \\
\text { presupuesto. }\end{array}$ \\
\hline $\mathrm{h} / \mathrm{m}^{2}$ & Ratio que relaciona el tiempo invertido y la superficie del proyecto. \\
\hline
\end{tabular}


Tabla 5. Acciones de mejora.

\begin{tabular}{|l|}
\hline \multicolumn{1}{|c|}{ Acciones de mejora } \\
\hline - Mejorar la coordinación entre las diferentes ingenierías, \\
fomentando una mayor comunicación y su incorporación \\
temprana al proyecto.
\end{tabular}

\subsection{Propuesta de nuevo flujo de trabajo}

El nuevo flujo de trabajo propuesto tiene como objetivo la mejora de la coordinación y comunicación de los agentes involucrados, para mejorar la productividad y la calidad del producto final y ha sido concebido para el método de desarrollo de proyecto tradicional, Proyecto-Licitación-Ejecución, ya que es al que debe adaptarse la empresa dadas las características de su mercado.

La explicación siguiente se centra exclusivamente en el flujo de trabajo una vez realizada la contratación. Se presupone redactado previamente un BEP, estableciendo las relaciones contractuales entre los agentes intervinientes, así como el uso de un Entorno de Datos Común para el intercambio de información, pero no se aborda su redacción dentro del flujo de trabajo, que está acotado únicamente al proceso de redacción del proyecto.

El flujo se divide en 5 etapas: Verificación, Reunión, Proceso de modelado, Documentación del proyecto y Control de Calidad.

\subsubsection{Verificación}

Una vez recibida la documentación del proyecto, se comprueba mediante checklist que está completa. En caso de detectar alguna carencia, se solicita al promotor que la solvente. Completada la documentación, se formaliza oficialmente el comienzo del modelado del proyecto.

\subsubsection{Reunión de Inicio}

El flujo de trabajo comienza convocando a asistir a una reunión de inicio a todos los agentes involucrados en el proceso de redacción del proyecto. Generalmente, se convoca al arquitecto responsable del proyecto y a los ingenieros responsables de la estructura y de las instalaciones, así como, si fuese el caso, a cualquier otro técnico responsable de redactar cualquier otra documentación aneja al proyecto (geólogo, coordinador de seguridad y salud, técnico de calidad y/o medioambiente, etc.). Si se conoce en este momento el/ los constructor(es) que va(n) a ejecutar la obra, también se le(s) convoca. También asisten los encargados de modelar el proyecto, en el caso de que no sea el propio arquitecto el responsable de ello.

Esta reunión es el inicio del LPS que va a dirigir la planificación y coordinación de toda la fase de redacción del proyecto. Por lo tanto, en esta primera reunión se lleva a cabo una PullSession entre todos los asistentes.

Además, para facilitar las labores de seguimiento, una de las pautas que se dan es que la duración de las actividades pro- puestas debe estar comprendida entre 1 y 2 semanas. Esta duración, que se convierte en referencia para otros proyectos, es susceptible de variar en la aplicación del nuevo procedimiento a otros proyectos en función de su complejidad y tamaño.

\section{La Pull-Session tiene como objetivos:}

1. Análisis de la viabilidad técnica. Se estudian las interferencias entre los elementos estructurales y las instalaciones en cuanto a la ubicación de pilares, límites de forjados, ubicación y tamaño de patinillos. Puede incluso plantearse la necesidad de llevar a cabo un análisis estructural y/o de las instalaciones a modo de comprobación. También se analizan las soluciones constructivas y el cumplimiento de la normativa técnica de aplicación.

2. Asignación de plazos a las tareas. En la figura 4 se indican unas duraciones estimadas de las actividades basadas en la experiencia adquirida en proyectos anteriores. No obstante, en esta Pull-Session, cada participante se compromete a una duración determinada para la realización de sus tareas, siempre con el esquema de la figura como referencia. De esta manera, se logra establecer un flujo de trabajo continuo en el que los plazos, rendimientos y tareas se establecen en función de la complejidad del proyecto, teniendo como objetivo principal mejorar la productividad del proceso de modelado BIM.

Tras esta reunión de inicio, cada participante es conocedor de las tareas que debe llevar a cabo y del plazo del que dispone para ello. El equipo redactor comienza ahora su análisis interno del proyecto, más exhaustivo que el anterior, con la finalidad de identificar no sólo los problemas potenciales, errores, omisiones que pueda contener sino también las mejoras posibles antes de comenzar con el proceso de modelado, evitando así generar re-trabajos innecesarios en fases posteriores.

\subsubsection{Proceso de modelado}

Se divide en tres fases:

Fase 1. Principales volúmenes y espacios

Una vez finalizado el análisis anterior, comienza el proceso de modelado BIM. En la primera fase se modelan los volúmenes y espacios principales del proyecto, lo que posibilita la realización de las siguientes tareas:

- Análisis del comportamiento energético del edificio. Se asegura el cumplimiento de los requisitos mínimos exigidos. En caso de ser necesario modificar alguna de las soluciones constructivas adoptadas, aún se está a tiempo de proceder a ello. Esta labor es realizada por un miembro del equipo redactor. Si se concluye que es necesario algún cambio o modificación se realiza un análisis y una propuesta de solución al proyectista para su validación o bien, para que remita una alternativa a la propuesta de modificación propuesta.

- Envío del modelo resultante a los colaboradores. De esta manera, el equipo encargado de proyectar las instalaciones y estructura comienzan a trabajar coordinadamente a partir de una única fuente de información, es decir, sobre el modelo BIM del edificio desarrollado siguiendo las direc- 
trices y criterios adoptados en la reunión de inicio, trabajando de forma colaborativa en un entorno común de datos e información.

\section{Fase 2. Desarrollo completo del modelo BIM}

Se desarrolla el modelo BIM del proyecto al nivel LOD especificado en el BEP. El otro miembro integrante del equipo redactor continúa con el desarrollo del modelo BIM hasta completarlo, añadiendo la información necesaria para poder obtener las mediciones del proyecto.

Fase 3. Cambios y mejoras propuestas por las ingenierías

Finalizadas las fases 1 y 2, se lleva a cabo la última fase del proceso de modelado BIM que consiste en introducir los cambios y mejoras propuestos y/o establecidos por las ingenierías (ampliación o ajuste del tamaño de patinillos, modificación del aislamiento de cerramientos, etc.).

\subsubsection{Documentación modelo BIM}

La última tarea, consistente en documentar el modelo BIM y redactar el resto de la documentación del proyecto, se lleva a cabo una vez finalizado el proceso de modelado.

Se elaboran los planos a partir del modelo, se redacta la memoria del proyecto y se realiza el presupuesto a partir de las mediciones extraídas del modelo. Para ello, al equipo redactor se une la persona encargada de redactar el presupuesto, que revisa las mediciones y determina los precios que correspondan. Una vez finalizada toda la documentación, se envía el proyecto completo al promotor, dando por finalizado el procedimiento de trabajo.

\subsubsection{Control y mejora continua del proceso}

Los tiempos invertidos en cada una de las tareas se controlan mediante indicadores de control y medidas de calidad que permiten analizar la productividad en el proceso de modelado BIM del proyecto de edificación.

Durante todo el flujo de trabajo, el contacto con el resto de agentes implicados es constante, por ser esencialmente necesario para resolver cualquier contingencia, aumentando la capacidad de reacción del equipo y permitiendo validar en menos tiempo las soluciones necesarias.

Así mismo, se establecen controles y seguimientos visuales de las tareas mediante la herramienta KANBAN, con tableros físicos en los entornos de trabajo y herramientas virtuales como Trello para la colaboración entre empresas.

\subsection{Resultados obtenidos}

La tabla 6 muestra un resumen de los proyectos analizados en el caso de estudio. Los proyectos 1-2-3 son realizados con el flujo de trabajo original de la empresa, mientras que los proyectos 4-5-6 se ejecutan con el nuevo flujo propuesto. Por sus características, los proyectos 1-2 son comparables al 4-5, mientras que el 3 se compara con el 6. Las figuras 2 y 3 muestran los principales resultados obtenidos con la aplicación del modelo LC-BIM, destacando la reducción de horas invertidas en modificaciones y cambios.

Como se observa, la aplicación del nuevo flujo supone una mejora real en la productividad en la redacción de proyectos de edificación, suponiendo un ahorro medio del $40 \%$ del tiempo empleado. Además, la calidad del trabajo realizado

Tabla 6. Resumen resultados obtenidos.

\begin{tabular}{|c|c|c|c|c|c|c|}
\hline & Proyecto 1 & Proyecto 2 & Proyecto 3 & Proyecto 4 & Proyecto 5 & Proyecto 6 \\
\hline Superficie & $1.200 \mathrm{~m}^{2}$ & $1.140 \mathrm{~m}^{2}$ & $2.247 \mathrm{~m}^{2}$ & $3.200 \mathrm{~m}^{2}$ & $3.800 \mathrm{~m}^{2}$ & $3.400 \mathrm{~m}^{2}$ \\
\hline Presupuesto & $2.415 .582 €$ & $1.300 .000 €$ & $1.350 .000 €$ & $1.875 .000 €$ & $2.150 .000 €$ & 1.820.00o€ \\
\hline Nivel complejidad & Alto & Alto & Bajo & Alto & Alto & Medio \\
\hline h/modelado & $125 \mathrm{~h}$ & $42 \mathrm{~h}$ & $53 \mathrm{~h}$ & $38 \mathrm{~h}$ & $13 \mathrm{~h}$ & $33 \mathrm{~h}$ \\
\hline $\begin{array}{l}\text { h/cam.modelo }+\mathbf{h} / \\
\text { cambios }\end{array}$ & $96 \mathrm{~h}$ & $85 \mathrm{~h}$ & 73h & $38 \mathrm{~h}$ & $29 \mathrm{~h}$ & $26 \mathrm{~h}$ \\
\hline \%new & $16,72 \%$ & $17,42 \%$ & $55,32 \%$ & $31,25 \%$ & $16,67 \%$ & $36,36 \%$ \\
\hline \%mod & $24,96 \%$ & $39,39 \%$ & $24,47 \%$ & $25,39 \%$ & $35,96 \%$ & $29,87 \%$ \\
\hline horas totales & $475 \mathrm{~h}$ & $435 \mathrm{~h}$ & $279 \mathrm{~h}$ & $249 \mathrm{~h}$ & $334 \mathrm{~h}$ & $17 \mathrm{Oh}$ \\
\hline $\mathbf{h} / \mathbf{m}^{2}$ & $0,40(24 \mathrm{~min})$ & 0,38 (23 min) & $0,12(8 \mathrm{~min})$ & $0,08(5 \mathrm{~min})$ & $0,08(5 \mathrm{~min})$ & o,o5 (3 min) \\
\hline
\end{tabular}

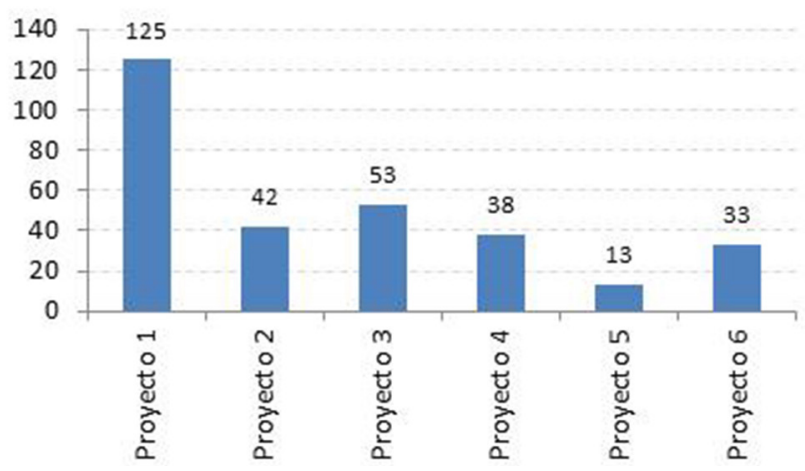

Figura 2. Horas/Modelado por proyecto.

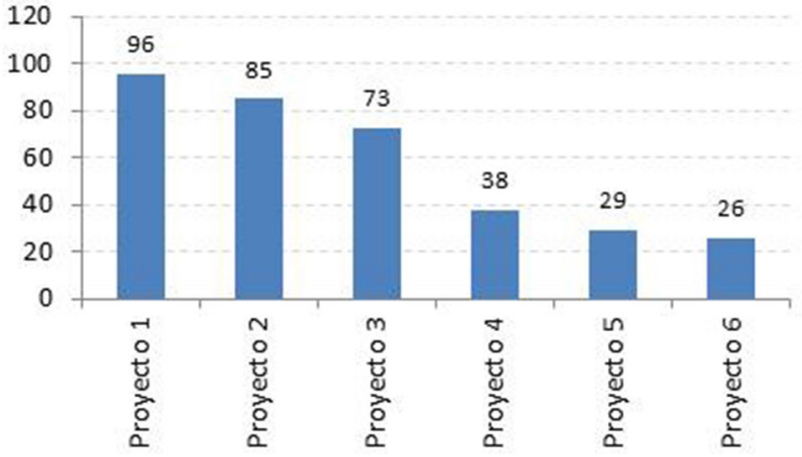

Figura 3. Horas/Cambios por proyecto. 
mejora, reduciendo un 65\% las horas empleadas en modificaciones y cambios durante el proceso.

\section{DISCUSIÓN}

Con los resultados obtenidos, se procede a la validación del modelo propuesto, mediante el uso de entrevistas a expertos como método principal, apoyado en la encuesta-cuestionario.

Se realizó un total de 12 entrevistas con profesionales expertos en la materia y con un rol importante en el sector y paralelamente, una encuesta a la que respondieron 272 encuestados. Los comentarios y aspectos de mejora del modelo se recogen en la tabla 7 .

Tras introducir las mejoras propuestas, se obtuvo el modelo final de la figura 4.

Tabla 7. Mejoras al modelo propuesto.

\begin{tabular}{|l|}
\hline \multicolumn{1}{|c|}{ Problemas identificados } \\
\hline Fomentar una mayor comunicación y más fluida. \\
\hline Determinar cómo se relaciona el modelo con el BEP. \\
\hline Introducir cuanto antes la participación de los agentes intervinientes. \\
\hline
\end{tabular}

\section{CONCLUSIÓN}

A partir de una revisión bibliográfica sobre las ventajas y beneficios del uso conjunto de LC y BIM, se ha elaborado un modelo LC-BIM para la redacción de proyectos de edificación. El modelo propuesto está adaptado método de desarrollo de proyectos tradicional (Proyecto-Licitación-Ejecución) imperante en el sector. Requisitos previos para su aplicación son la elaboración de un BEP y el establecimiento de un Entorno de Datos Comunes (Common Data Environment, CDE) para ser usado posteriormente por todos los agentes implicados.

Posteriormente se ha realizado un estudio comparando 6 proyectos de una misma empresa, 3 realizados con el flujo de trabajo original de la misma y 3 con el flujo propuesto, obteniendo como resultados una mejora real tanto de la productividad como de la calidad de los proyectos realizados con el nuevo flujo.

Como última etapa de la investigación, se ha procedido a la validación del modelo LC-BIM mediante el uso de técnicas cualitativas (entrevistas) y cuantitativas (encuestas), a resultas de la cual se han podido determinar las mejoras finalmente introducidas en el modelo final LC-BIM (figura 4).

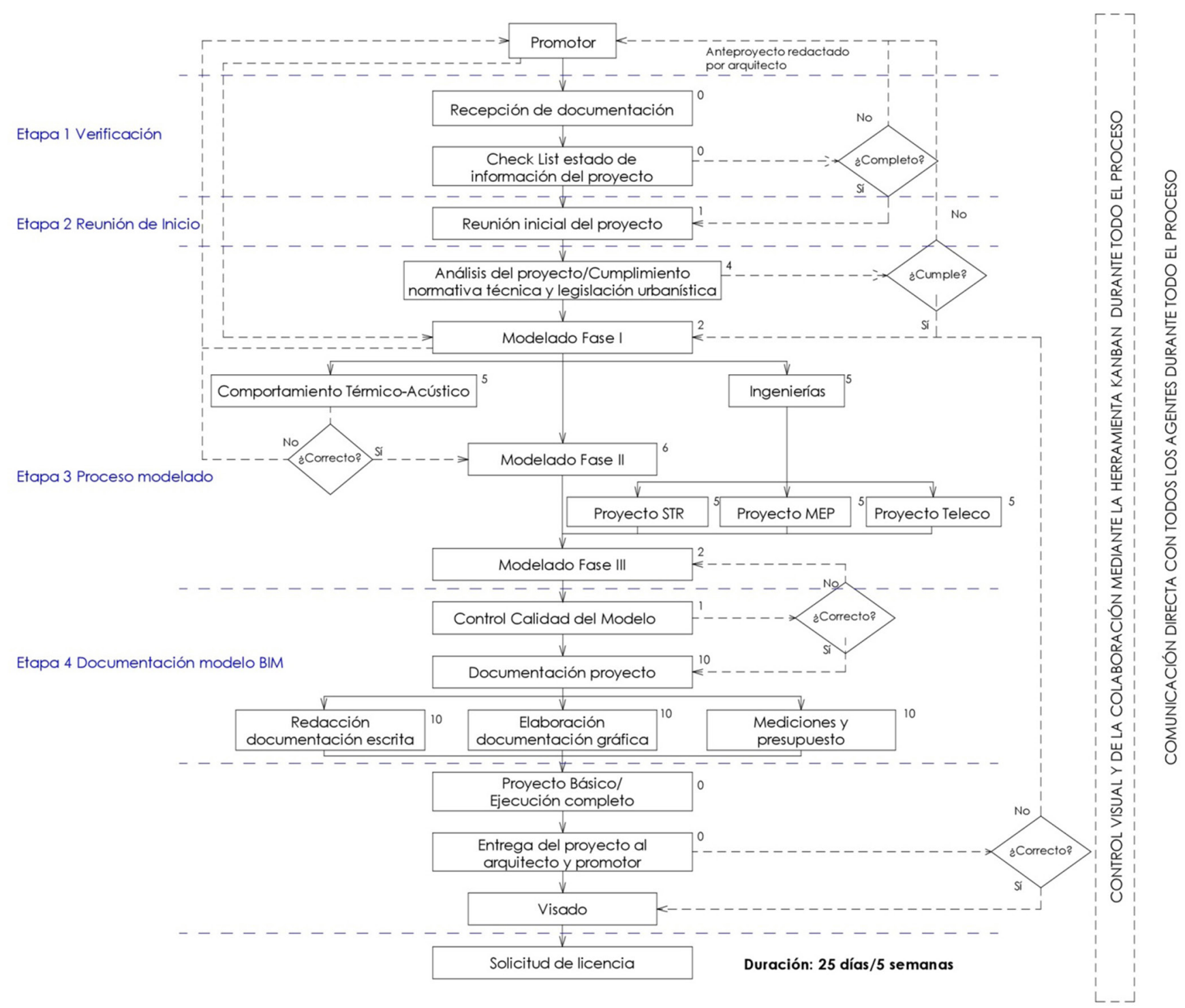

Figura 4. Modelo LC-BIM. 
Este modelo logra mejorar la comunicación y colaboración entre todos los agentes implicados en el desarrollo del proyecto de ejecución. El uso de BIM permite la mayor y mejor comprensión del proyecto a los agentes implicados, logrando realizar un mejor producto, y combinando su uso con Last Planner, se consigue establecer una planificación realista de hitos y entregas, que unido a lo visual que resulta su seguimiento con la herramienta Kanban, permite mejorar la productividad de todo el proceso.

No obstante, este modelo es susceptible de mejoras. El principal reto de futuras investigaciones es su modificación para adaptarlo a métodos de desarrollo de proyectos más colabo- rativos, como el Integrated Project Delivery (IPD), posibilitando obtener aún mayores beneficios de la combinación de LC y BIM.

El sector de la Construcción debe cambiar para mejorar la productividad, siguiendo el camino que han seguido otros sectores como la industria o el del automóvil. Se debe dar un nuevo paradigma de la construcción, orientado hacia la filosofía Lean, y cuya principal herramienta de digitalización sea BIM. El modelo LC-BIM para la redacción de proyectos de edificación es la demostración práctica de que todas aquellas mejoras que se intuían podían darse combinando el uso de la filosofía Lean y BIM son realidad.

\section{REFERENCIAS}

(1) Bhatla, A., Leite, F. (2012). Integration Framework of BIM with the Last Planner System. En 20th Annual Conference of the International Group for Lean Construction, San Diego.

(2) Sacks, R., Koskela, L., Dave, B.A., \& Owen, R. (2010). Interaction of lean and building information modeling in construction. Journal of construction engineering and management, 136(9): 968-980. https://doi.org/10.1061/(ASCE) CO.1943-7862.0000203

(3) Hamdi, O., Leite, F. (2012). BIM and Lean interactions from the BIM capability maturity model perspective: a case study. En 2oth Annual Proceeding of the International Group for Lean Construction, San Diego.

(4) Clemente, J., Cachadinha, N. (2013). BIM-Lean Synergies in the Management on MEP Works in Public Facilities of Intensive Use - a Case Study. En Annual Conference of the International Group for Lean Construction (pp. 751-759), Fortaleza.

(5) Latorre, A. (2015). Filosofía Lean en la construcción. Valencia: Universitat Politècnica de València. https://riunet.upv. es/handle/10251/50732

(6) Salmon, J.L. (2009). The Legal Revolution in Construction. Journal of Building Information Modeling (JBIM), (Spring): 18-19. Recuperado de https://www.brikbase.org/content/legal-revolution-construction.

(7) Tauriainen, M., Marttinen, P., Dave, B., Koskela, L. (2016). The Effects of BIM and Lean Construction on Design Management Practices. Procedia Engineering, 164: 567-574. https://doi.org/10.1016/j.proeng.2016.11.659

(8) Mollasalehi, A. (2016). Development of an experimental waste framework based on BIM/lean concept in construction design. En 24th Annual Conference of the International Group for Lean Construction (pp. 193-202), Boston. Recuperado de http://usir.salford.ac.uk/43968/

(9) Nascimento, D.L. de M., Sotelino, E.D., Caiado, R.G.G., Ivson, P., Faria, P.S. (2017). Synergy between principles of lean thinking and BIM functionalities in interdisciplinarity of management in industrial plants. Journal of Lean Systems, 2(4): 80-105. Recuperado de http://ojs.sites.ufsc.br/index.php/lean/article/view/1758

(10) Fakhimi, A.H., Majrouhi Sardroud, J., Azhar, S. (2016). How Can Lean, IPD and BIM Work Together? En Proceedings of the 33rd ISARC (pp. 67-75), Auburn, AL, USA. https://doi.org/10.22260/ISARC2016/o009

(11) Zhang, X., Azhar, S., Nadeem, A., Khalfan, M. (2018). Using Building Information Modelling to achieve Lean principles by improving efficiency of work teams. International Journal of Construction Management, 18(4):293-300. https:// doi.org/10.1080/15623599.2017.1382083

(12) Mandujano, M.G., Alarcón, L.F., Kunz, J., Mourgues, C. (2016). Identifying waste in virtual design and construction practice from a Lean Thinking perspective: A meta-analysis of the literature. Revista de la Construcción, 15(3): 107-118. https://doi.org/10.4067/So718-915X2016000300011

(13) Alvarenga, T.W., da Silva, E.N., Mello, L.C.B. de B. (2017). BIM and Lean Construction: The Evolution Obstacle in the Brazilian Civil Construction Industry. Engineering, Technology \& Applied Science Research, 7(5): 1904-1908. Recuperado de http://etasr.com/index.php/ETASR/article/view/1278

(14) Avila Díaz, J.O., Quintana Pulido, N. (2015). Simulación de Eventos Discretos y Líneas de Balance, Aplicadas al Mejoramiento del Proceso Constructivo del Edificio Universidad Ciudad PUJ. Bogotá: Pontificia Universidad Javeriana, Facultad de Ingeniería. Recuperado de https://repository.javeriana.edu.co/handle/10554/12693

(15) Dave, B., Kubler, S., Pikas, E., Holmström, J., Singh, V., Främling, K., Koskela, L. (29-31 July 2015). Intelligent Products: Shifting the Production Control Logic in Construction (With Lean and BIM). En 23th Annual Conference of the International Group for Lean Construction, Perth, Australia. Recuperado de https://aaltodoc.aalto.fi/handle/123456789/17643

(16) Rischmoller, L., Alarcón, L.F., Koskela, L. (2006). Improving Value Generation in the Design Process of Industrial Projects Using CAVT. Journal of Management in Engineering, 22(2): 52-60. https://doi.org/10.1061/(ASCE)O742$597 \mathrm{X}(2006) 22: 2(52)$

(17) Khanzode, A., Fischer, M., Reed, D., Ballard, G. (2006). A guide to applying the principles of virtual design \& construction $(V D C)$ to the lean project delivery process. CIFE, Stanford University. Recuperado de https://stacks.stanford.edu/ file/druid:bc98obz5582/WPo93.pdf

(18) Álvarez, M.Á., Bucero, A., Pampliega, C.J. (2017). Integrated project delivery, an alternative to the usual form of construction work in Spain = Métodos colaborativos, un cambio para la construcción en España. Building \& Management, 1(3): 30-36. https://doi.org/10.20868/bma.2017.3.3656 
(19) Galsworth, G.D. (1997). Visual systems: harnessing the power of the visual workplace. New York: American Management Association.

(20) Sacks, R., Dave, B., Koskela, L., Owen, R. (2009). Analysis framework for the interaction between lean construction and building information modeling. En 17th Annual Conference of the International Group for Lean Construction, National Pingtung University of Science and Technology, LCI-Taiwan and LCI-Asia, Taipei.

(21) Dave, B., Boddy, S., Koskela, L. (2013). Challenges and opportunities in implementing lean and BIM on an infrastructure project. En 21th Annual Conference of the International Group for Lean Construction, Fortaleza, Brazil. Recuperado de http://iglc.net/Papers/Details/888/pdf

(22) Oskouie, P., Gerber, D.J., Alves, T., Becerik-Gerber, B. (2012). Extending the interaction of building information modeling and lean construction. En Proceedings for the 2oth Annual Conference of the International Group for Lean Construction. Recuperado de http://citeseerx.ist.psu.edu/viewdoc/download?doi=10.1.1.705.3874\&rep=rep1\&type=pdf

(23) Kjartansdóttir, I.B. (2011). BIM Adoption in Iceland and Its Relation to Lean Construction. Reykjavík: Reykjavík University. Recuperado de https://skemman.is/bitstream/1946/10907/1/Ingibj\%C3\%B6rgBirnaKj.Tilb\%C3\%BAi\%C3\%Bo040112.pdf

(24) Dave, B. (2013). Developing a construction management system based on lean construction and building information modelling (Tesis doctoral). University of Salford.

(25) Dave, B., Koskela, L.J., Kiviniemi, A.O., Tzortzopoulos Fazenda, P. and Owen, R.L. (2013). Implementing lean in construction: Lean construction and BIM, Manual. London: CIRIA. Recuperado de http://usir.salford.ac.uk/28451/

(26) Olivares Belmar, K.G. (2017). Exploración de un marco de planificación LEAN-BIM: un sistema last planner y BIM basado en 2 casos de estudio. Universidad Andrés Bello. Recuperado de http://repositorio.unab.cl/xmlui/ handle/ria/3524.

(27) Toledo, M., Olivares, K., González, V. (2016). Exploration of a Lean-BIM Planning Framework: A Last Planner System and BIM-Based Case Study, En 24th Annual Conference of the International Group for Lean Construction(pp. 3-12), Boston, MA, USA. Recuperado de https://iglcstorage.blob.core.windows.net/papers/attachment-bebfd63e-3f20-409b-9f85-dc73ad8bb5d8.pdf

(28) Tauriainen, M., Marttinen, P., Dave, B., Koskela, L. (2016). BIM and Lean Construction Change Design Management Practices. En Creative Construction Conference 2016 (pp. 668-673), Budapest. Recuperado de http://2016.creativeconstruction-conference.com/proceedings/CCC2016_104_Tauriainen.pdf

(29) Villa, V., Di Giuda, G.M., Giana, P.E., Tagliabue, L.C., Ciribini, A.L.C. (2017). Lean construction applied to a BIM process: how to control point attribution. En MEAT tender process, Tema: Technology, Engineering, Materials and Architecture. 3: 35-44. https://doi.org/10.17410/tema.v3i1.121

(30) Saieg, P., Sotelino, E.D., Nascimento, D., Caiado, R.G.G. (2018). Interactions of Building Information Modeling, Lean and Sustainability on the Architectural, Engineering and Construction industry: A systematic review. Journal of Cleaner Production, 174: 788-806. https://doi.org/10.1016/j.jclepro.2017.11.030 